PROCEEDINGS OF THE

AMERICAN MATHEMATICAL SOCIETY

Volume 132, Number 7, Pages 1987-1995

S 0002-9939(03)07335-0

Article electronically published on December 19, 2003

\title{
A NOTE ON THE SUPPORT OF A SOBOLEV FUNCTION ON A $k$-CELL
}

\author{
W. K. ZIEMER
}

(Communicated by David Preiss)

\begin{abstract}
It is shown that a $k$-cell (the homeomorphic image of a closed ball in $\left.\mathbb{R}^{k}\right)$ in $\mathbb{R}^{n}, 1 \leq k<n$, cannot support a function in $W^{1, p}\left(\mathbb{R}^{n}\right)$ if $p>\left[\frac{k+1}{2}\right]$, the greatest integer in $(k+1) / 2$.
\end{abstract}

\section{INTRODUCTION}

In this paper we investigate the question of determining whether the homeomorphic image of a $k$-dimensional closed ball in $\mathbb{R}^{n}, 1 \leq k<n$, a $k$-cell, can support a Sobolev function $f \in W^{1, p}\left(\mathbb{R}^{n}\right)$. Since a $k$-cell is nowhere dense in $\mathbb{R}^{n}$, it is natural to first inquire whether a compact, nowhere dense set can support a Sobolev function. Of course, this question is only of interest when the set has positive Lebesgue measure. For the case $p>n$, the answer is obvious, since any function of $W^{1, p}\left(\mathbb{R}^{n}\right)$ has a continuous representative in $\mathbb{R}^{n}$, and a nonzero continuous function cannot have its support on a nowhere dense compact set. However, for the case $1<p \leq n$, Polking [Pol72] Theorem 4] showed that there is a nonzero element of $W^{1, p}\left(\mathbb{R}^{n}\right)$ that does have nowhere dense compact support. A characterization of nowhere dense sets that can support $W^{1, p}\left(\mathbb{R}^{n}\right)$ functions in terms of capacity is given in [AH96. Theorem 11.3.2]. The existence of homeomorphisms that carry sets of Lebesgue measure zero into sets of positive measure is well known. Besicovitch Bes50] constructed a homeomorphism from $\mathbb{R}^{2}$ to $\mathbb{R}^{3}$ that carries null sets onto sets of positive measure. In [Pon87, a homeomorphism in $W^{1, q}\left(\mathbb{R}^{n} ; \mathbb{R}^{n}\right)$, with $q<n$, was constructed carrying null sets into sets of positive Lebesgue measure. The question we investigate in this paper is whether a $k$-cell in $\mathbb{R}^{n}, 0<k<n$, can support a Sobolev function $u \in W^{1, p}\left(\mathbb{R}^{n}\right)$. The complete answer to this question remains an open problem. Bagby and Gauthier [BG98] proved that for $n>k>0$ and $p>\max (1, k-1)$, only the zero function in $W^{1, p}\left(\mathbb{R}^{n}\right)$ has its support contained in a $k$-cell. Our contribution to this question is to offer an improvement of this result for $n \geq 3$. In Theorem 5 of this paper it is shown that the Bagby-Gauthier result remains true by requiring $p>\left[\frac{k+1}{2}\right]$ where $\left[\frac{k+1}{2}\right]$ denotes the greatest integer in $\frac{k+1}{2}$. The main ingredient of the proof is that under these restrictions on $p$, if $u \in W^{1, p}\left(\mathbb{R}^{k+1}\right)$ is not identically zero, then $u$ has a representative that is defined, continuous and strictly positive (or negative) on a pair of linked spheres of dimension $\left[\frac{k+1}{2}\right]$ and $\left[\frac{k}{2}\right]$; see Definition [1.

Received by the editors May 3, 2001 and, in revised form, March 11, 2003.

2000 Mathematics Subject Classification. Primary 46E35. 


\section{Preliminaries}

The Lebesgue measure of a set $E \subset \mathbb{R}^{n}$ is denoted by $|E|$, its $s$-dimensional Hausdorff measure by $H^{s}(E)$, and its $p$-capacity by $\gamma_{p}(E)$. We refer the reader to MZ97, Section 2.1] for the definitions of $p$-capacity, its comparison to Hausdorff measure, and its relationship to functions in the Sobolev class $W^{1, p}$. In particular, we recall that

$$
\gamma_{p}(E)=0 \text { if and only if } H^{n-p+\varepsilon}(E)=0 \text { for all } \varepsilon>0 \text { and } 1 \leq p \leq n .
$$

The restriction of a function $u$ to a set $E$ is denoted by $u\left\llcorner E\right.$. With $\Omega \subset \mathbb{R}^{n}$ an open set and $n \geq 1$, the Sobolev space $W^{1, p}(\Omega), p \geq 1$, consists of those functions $u \in L^{p}(\Omega)$ for which the first-order distributional partial derivatives of $u$ also belong to $L^{p}(\Omega)$. The norm on $W^{1, p}(\Omega)$ is given by

$$
\|u\|_{1, p ; \Omega}=\left(\sum_{k=0}^{n} \int_{\Omega}\left|D^{k} u\right|^{p} d x\right)^{1 / p} .
$$

An alternate definition of the Sobolev space is furnished by the fact that $C^{\infty}(\Omega) \cap$ $\left\{u:\|u\|_{1, p ; \Omega}<\infty\right\}$ is dense in $W^{1, p}(\Omega)$. A sequence of functions that converges except on a set of $\gamma_{p}$ zero is said to converge $p$-q.e. A function $u$ is called $p$ quasicontinuous if for each $\varepsilon>0$, there exists an open set $U \subset \mathbb{R}^{n}$ with $\gamma_{p}(U)<\varepsilon$ such that $u L \mathbb{R}^{n} \backslash U$ is continuous. Any function $u \in W^{1, p}\left(\mathbb{R}^{n}\right)$ has a representative that is $p$-quasicontinuous. Indeed, the pointwise limit of a suitable subsequence of smooth functions $\left\{u_{k}\right\}$ that converge strongly to $u$ in $W^{1, p}$ defines a p-quasicontinuous representative; cf. [MZ97, Lemma 2.19]. Throughout, we will employ the notation $\mathbf{u}$ (boldface $u$ ) to denote a $p$-quasicontinuous representative of $u \in W^{1, p}\left(\mathbb{R}^{n}\right)$ and $B_{x}^{n}(r)$ to denote the open ball in $\mathbb{R}^{n}$ with center $x$ and radius $r$. Recall that an arbitrary $u \in L^{p}\left(\mathbb{R}^{n}\right)$ has an $L^{p}$-Lebesgue point almost everywhere; that is,

$$
\lim _{r \rightarrow 0} \frac{1}{\left|B_{a}^{n}(r)\right|} \int_{B_{a}^{n}(r)}|u(x)-u(a)|^{p} d x=0
$$

for almost all $a \in \mathbb{R}^{n}$. When $u \in W^{1, p}\left(\mathbb{R}^{n}\right)$, this limit is zero for all $a$ in the complement of a $\gamma_{p}$ null set. If $a$ is a Lebesgue point for $u$ and if $\left\{u_{k}\right\}$ is taken as the standard mollifiers of $u$, then $u_{k}(a) \rightarrow u(a)$. We will use the notation $\bar{u}(a, r)$ to denote the integral average of $u$ over the the ball $B_{a}^{n}(r)$, and $\bar{u}(a):=\lim _{r \rightarrow 0} \bar{u}(a, r)$ when the limit exists. Likewise, we let $\overline{\nabla u}(a)$ denote the value of $\nabla u$ in terms of the limit of its integral averages at $a$.

Throughout, we will assume that $1 \leq p \leq n$ since our problem becomes trivial if $p>n$. We will make extensive use of the "coarea formula", stated below.

Theorem 1 ([Fed59, Theorem 3.1]). If $X$ and $Y$ are separable Riemannian manifolds of class 1 of respective dimensions $m$ and $k, m \geq k$, and $f: X \rightarrow Y$ is a Lipschitzian map, then

$$
\int_{X} g(x) J f(x) d H^{m}(x)=\int_{Y} \int_{f^{-1}(y)} g(x) d H^{m-k}(x) d H^{k}(y)
$$

whenever $g: X \rightarrow \mathbb{R}^{1}$ is $H^{m}$ integrable. Here, Jf $(x)$ denotes the square root of the sum of the squares of the determinants of the $k \times k$ minors of the differential of $f$ at $x$. 
We will not need the full strength of Federer's coarea formula, but merely the case when $X$ and $Y$ are subsets of Euclidean space.

\section{LINKED SPHERES IN $\mathbb{R}^{n}$}

Definition 1. With $S^{k}$ denoting the standard $k$-sphere in $\mathbb{R}^{k+1}$, let $\Sigma_{1}^{k}:=h_{1}\left(S^{k}\right)$ and $\Sigma_{2}^{n-1-k}:=h_{2}\left(S^{n-1-k}\right)$ be the images of disjoint topological embeddings, $h_{1}, h_{2}$, of $S^{k}$ and $S^{n-1-k}$ into $\mathbb{R}^{n}$. The linking number of $\Sigma_{1}^{k}$ and $\Sigma_{2}^{k}$ is defined as the topological degree of the mapping

$$
S^{k} \times S^{n-1-k} \stackrel{f}{\longrightarrow} S^{n-1}
$$

defined by $f(x, y)=\frac{h_{1}(x)-h_{2}(y)}{\left|h_{1}(x)-h_{2}(y)\right|}$; see [Hir76] or [Rol76].

Remark 1. Recall that the topological degree is defined to be that integer, $\operatorname{deg}(f)$, so that the induced homomorphism of homology groups, $f_{*}: H_{n-1}\left(S^{k} \times S^{n-1-k}\right) \rightarrow$ $H_{n-1}\left(S^{n-1}\right)$, is given by multiplication by $\operatorname{deg}(f)$. Note that both homology groups are isomorphic to $\mathbb{Z}$. Recall also that if $f$ is smooth, then $\operatorname{deg}(f)=\sum_{x \in f^{-1}(y)} \mathcal{J} f(x)$ where $y$ is a regular value of $f$, and where $\mathcal{J} f(x)$ denotes the Jacobian of $f$ at $x$.

Theorem 2. Let $\bar{B}^{n-1}$ be a closed ball in $\mathbb{R}^{n-1}$ and suppose that $h: \bar{B}^{n-1} \rightarrow \mathbb{R}^{n}$ is an embedding, with disjoint $\Sigma_{1}^{k}, \Sigma_{2}^{n-1-k} \subset h\left(B^{n-1}\right)$. Then the linking number of $\Sigma_{1}^{k}$ and $\Sigma_{2}^{n-1-k}$ is 0 .

Proof. The mapping $f$ in (3.1) can be factored as $f=f_{2} \circ f_{1}$ where

$$
f_{1}: S^{k} \times S^{n-1-k} \rightarrow\left(h\left(\bar{B}^{n-1}\right) \backslash \Sigma_{2}^{n-1-k}\right) \times \Sigma_{2}^{n-1-k}
$$

and

$$
f_{2}:\left(h\left(\bar{B}^{n-1}\right) \backslash \Sigma_{2}^{n-1-k}\right) \times \Sigma_{2}^{n-1-k} \rightarrow S^{n-1} .
$$

Let $H_{i}(K)$ denote the $i t h$ homology group of $K$. Recalling the Künneth theorem, Mas91, Section XI.4, Theorem 4.1], and the fact that $H_{i}\left(S^{k}\right)$ and $H_{i}\left(S^{k} \backslash S^{j}\right)$ are torsion free, we have that

$$
\begin{aligned}
& H_{q}(\left.\left(h\left(\bar{B}^{n-1}\right) \backslash \Sigma_{2}^{n-1-k}\right) \times \Sigma_{2}^{n-1-k}\right) \\
& \quad=\sum_{j=0}^{q} H_{j}\left(h\left(\bar{B}^{n-1}\right) \backslash \Sigma_{2}^{n-1-k}\right) \otimes H_{q-j}\left(\Sigma_{2}^{n-1-k}\right) .
\end{aligned}
$$

Since $h\left(\bar{B}^{n-1} \backslash \Sigma_{2}^{n-1-k}\right)$ is homeomorphic to $\bar{B}^{n-1} \backslash h^{-1}\left(\Sigma_{2}^{n-1-k}\right)$, the complement of an embedded $(n-1-k)$-sphere, we obtain the following homology groups: for $k>1$,

$$
H_{q}\left(h\left(\bar{B}^{n-1}\right) \backslash \Sigma_{2}^{n-1-k}\right)=\left\{\begin{array}{cc}
\mathbb{Z} & \text { when } q=0, k-1, \text { and } n-2, \\
0 & \text { otherwise }
\end{array}\right.
$$

and

$$
H_{q}\left(\Sigma_{2}^{n-1-k}\right)=\left\{\begin{array}{cc}
\mathbb{Z} & \text { when } q=0, \text { and } n-1-k \\
0 & \text { otherwise }
\end{array}\right.
$$

Therefore,

except when

$$
H_{q}\left(\left(h\left(\bar{B}^{n-1}\right) \backslash \Sigma_{2}^{n-1-k}\right) \times \Sigma_{2}^{n-1-k}\right)=0
$$

$$
q \in\{0, k-1, n-2, n-2-k, 2 n-3-k\}
$$


Consequently, $H_{n-1}\left(\left(h\left(\bar{B}^{n-1}\right) \backslash \Sigma_{2}^{n-1-k}\right) \times \Sigma_{2}^{n-1-k}\right)=0$ unless $2 n-3-k=n-1$; that is, if $k=n-2$. However, without loss of generality, it can be assumed that $k<n / 2$, and therefore $H_{n-1}\left(\left(h\left(\bar{B}^{n-1}\right) \backslash \Sigma_{2}^{n-1-k}\right) \times \Sigma_{2}^{n-1-k}\right)=0$ except when $n=3$. When $n=3$, the Jordan Curve Theorem can be applied to the curves $\Sigma_{1}^{k}$ and $\Sigma_{2}^{n-1-k}$ in $h\left(\bar{B}^{2}\right)$ to conclude that one of the curves is null homotopic in the complement of the other. Since the degree is a homotopy invariant, in this case the degree will be 0 as well.

\section{Quasicontinuous RePresentatives on SPHERES}

For $3 \leq m+2 \leq n$ and $x=\left(x_{1}, x_{2}, \ldots, x_{n}\right) \in \mathbb{R}^{n}$, we will write $x=\left(x^{\prime}, x^{\prime \prime}\right)$ where $x^{\prime}=\left(x_{1}, x_{2}, \ldots, x_{m+1}\right)$ and $x^{\prime \prime}=\left(x_{m+2}, \ldots, x_{n}\right)$. Let $Q: \mathbb{R}^{n} \rightarrow \mathbb{R}^{n-m-1}$ be defined as $Q(x)=x^{\prime \prime}$. Then $Q^{-1}\left(x^{\prime \prime}\right)$ is an $(m+1)$-dimensional "horizontal" affine space. Throughout, we will use the notation $S_{x}^{m}(r)$ to denote the $m$-sphere centered at $x \in \mathbb{R}^{n}$ of radius $r$ that is contained in $Q^{-1}\left(x^{\prime \prime}\right)$. Thus,

$$
\begin{aligned}
S_{x}^{m}(r) & =\left\{y \in Q^{-1}\left(x^{\prime \prime}\right):\left|y-x^{\prime}\right|=r\right\} \\
& =\left\{y \in \mathbb{R}^{n}: y=\left(y^{\prime}, x^{\prime \prime}\right),\left|y^{\prime}-x^{\prime}\right|=r\right\} .
\end{aligned}
$$

We will also consider spheres in planes orthogonal to $Q^{-1}\left(x^{\prime \prime}\right)$, using the familiar notation for spheres. Thus, for $b \in S_{a}^{m}(r)$ we will consider an $(n-m-1)$-sphere centered at $b$ in the $(n-m)$-plane orthogonal to $Q^{-1}\left(a^{\prime \prime}\right)$ that contains the line through $a$ and $b$; thus, for $b \in S_{a}^{m}(r)$ and $0<\rho<r$ we define

$$
S_{b}^{n-m-1}(\rho)=\left\{y \in \mathbb{R}^{n}:|y-b|=\rho, y^{\prime}=\alpha\left(b^{\prime}-a^{\prime}\right)+a^{\prime}, \alpha \in \mathbb{R}^{1}\right\} .
$$

It can be shown as a direct consequence of the definition that these spheres are linked. Also, see Gage81, introductory remark].

For any $a \in \mathbb{R}^{n}$, let $F_{a}: \mathbb{R}^{n} \rightarrow \mathbb{R}^{n-m}$ be defined as $F_{a}(x)=F_{a}\left(x^{\prime}, x^{\prime \prime}\right)=$ $\left(\left|x^{\prime}-a^{\prime}\right|, x^{\prime \prime}\right) \in \mathbb{R}^{n-m}$. Thus, for $z=\left(z_{1}, \ldots, z_{n-m}\right) \in \mathbb{R}^{n-m}$, we have

$$
\begin{aligned}
F_{a}^{-1}(z) & =\left\{y \in \mathbb{R}^{n}:\left|y^{\prime}-a^{\prime}\right|=z_{1}, y^{\prime \prime}=\left(z_{2}, \ldots, z_{n-m}\right)\right\} \\
& =S_{\left(a^{\prime}, z_{2}, \ldots, z_{n-m}\right)}^{m}\left(z_{1}\right) .
\end{aligned}
$$

It is not difficult to verify that $J F_{a}=1$ and that $F_{a}$ is Lipschitz with Lipschitz constant 1. Let $I_{r} \subset \mathbb{R}^{n-m}$ denote the cube in $\mathbb{R}^{n-m}$ of side length $r>0$ and center $(r, 0, \ldots, 0)$. Then $F_{a}^{-1}\left(I_{r}\right):=\bigcup_{w \in I_{r}} F_{a}^{-1}(w)$ defines a "rectangular torus". For example, if $n=3, m=1, a=0 \in \mathbb{R}^{3}$, and $I_{r}$ is the $r$ by $r$ square in the $(y, z)$-plane with center $(r, 0)$, then $F_{a}^{-1}\left(I_{r}\right)$ is the figure obtained by rotating $I_{r}$ about the $z$-axis.

Theorem 3. Let $u \in W^{1, p}\left(\mathbb{R}^{n}\right)$, let $m$ be an integer with $n \geq m+2 \geq 3, p>m$ and let $\mathbf{u}$ denote an arbitrary, but fixed, p-quasicontinuous representative of $u$ as determined by the pointwise limit of a suitable subsequence of smooth functions $u_{k}$ that converge strongly to $u$ in $W^{1, p}\left(\mathbb{R}^{n}\right)$. Then:

(i) $\mathbf{u}$ is continuous on $F_{a}^{-1}(w)$ for $H^{n-m}$-a.e. $w \in \mathbb{R}^{n-m}$.

(ii) If $a \in \mathbb{R}^{n}$ is an $L^{p}$-Lebesgue point for both $u$ and $|\nabla u|$ and if

$$
\bar{u}(a)>0,
$$

then there exists $R_{0}>0$ such that for every $0<r<R_{0}$ there exists an $H^{n-m}$ measurable set $E_{r} \subset I_{r}$ of positive $H^{n-m}$-measure such that $\mathbf{u}$ is continuous and positive on $F_{a}^{-1}(w)$ for $w \in E_{r}$. 
Proof. (i) Since $u \in W^{1, p}\left(\mathbb{R}^{n}\right)$, we know that $u$ is the strong limit of functions $u_{k} \in C^{\infty}\left(\mathbb{R}^{n}\right)$ and therefore, for each $\varepsilon>0$, there exists an open set $U_{\varepsilon} \subset \mathbb{R}^{n}$ and a subsequence such that $\gamma_{p}\left(U_{\varepsilon}\right)<\varepsilon$ and that the $u_{k}$ converge to $u$ uniformly on $\mathbb{R}^{n} \backslash U_{\varepsilon}$; cf. [MZ97, Lemma 2.19]. Choosing a sequence $\varepsilon_{j} \rightarrow 0$, we see that $\gamma_{p}(U)=0$ where $U:=\bigcap_{\varepsilon_{j}} U_{\varepsilon_{j}}$. Since $F_{a}$ is Lipschitz, $\gamma_{p}\left(F_{a}\left(U_{\varepsilon_{j}}\right)\right) \leq C \gamma_{p}\left(U_{\varepsilon_{j}}\right)<$ $C \varepsilon_{j}$, where $C=C(p, n)$, AH96, Theorem 5.2.1]. Let

$$
E:=\bigcap_{\varepsilon_{j}>0} F_{a}\left(U_{\varepsilon_{j}}\right) .
$$

Then $\gamma_{p}(E)=0$, so that $H^{n-p+\varepsilon}(E)=0$ for all $\varepsilon>0$, by (2.1). Since $p>m$, there exists $\varepsilon>0$ and $0<\alpha<1$ such that $n-p+\varepsilon=n-m-\alpha$, and therefore $H^{n-m-\alpha}(E)=0$. This, in turn, implies that $H^{n-m}(E)=0$. If $w \notin E$, then $w \notin F_{a}\left(U_{\varepsilon_{j}}\right)$ for some $j$, which implies that $F_{a}^{-1}(w) \cap U_{\varepsilon_{j}}=\emptyset$. Thus $\mathbf{u}$, the uniform pointwise limit of the $u_{k}$ on $\mathbb{R}^{n} \backslash U_{\varepsilon_{j}}$, is continuous on $F_{a}^{-1}(w)$ for $w \notin E$. That is, $\mathbf{u}$ is continuous on $F_{a}^{-1}(w)$ for $H^{n-m}$-a.e. $w \in \mathbb{R}^{n-m}$.

(ii) The proof is divided into three parts.

Step 1. For $H^{n-m}$-a.e. $w \in I_{r}, u_{w}:=u\left\llcorner F_{a}^{-1}(w)\right.$, we claim that

$$
\sup _{F_{a}^{-1}(w)}|\mathbf{u}| \leq C\left(\int_{F_{a}^{-1}(w)} r^{p-m}\left|\nabla\left(u_{w}\right)\right|^{p}+r^{-m}\left|u_{w}\right|^{p} d H^{m}\right)^{1 / p},
$$

with $C$ a constant. For this, observe that the co-area formula yields

$$
\begin{aligned}
\lim _{k \rightarrow \infty} & \int_{I_{r}} \int_{F_{a}^{-1}(w)}\left|\nabla u_{k}-\nabla u\right|^{p}+\left|u_{k}-u\right|^{p} d H^{m} d H^{n-m}(w) \\
& =\lim _{k \rightarrow \infty} \int_{F_{a}^{-1}\left(I_{r}\right)}\left(\left|\nabla u_{k}-\nabla u\right|^{p}+\left|u_{k}-u\right|^{p}\right) d H^{n} \\
& =0 .
\end{aligned}
$$

Thus there is a subsequence of the $u_{k}$ (still denoted as the full sequence) such that for $H^{n-m}$-a.e. $w \in I_{r}$,

$$
\lim _{k, l \rightarrow \infty} \int_{F_{a}^{-1}(w)}\left|\nabla u_{k}-\nabla u_{l}\right|^{p}+\left|u_{k}-u_{l}\right|^{p} d H^{m}=0 .
$$

This subsequence converges strongly to some element of $W^{1, p}\left(F_{a}^{-1}(w)\right)$, which we denote by

$$
u\left\llcorner F_{a}^{-1}(w) .\right.
$$

Since $u_{k} \rightarrow \mathbf{u}$ uniformly on $F_{a}^{-1}(w)$ for $w \notin E$, observe that $\mathbf{u} L F_{a}^{-1}(w)$ is a continuous representative of $u L F_{a}^{-1}(w)$. To ease notation, we will write $u_{w}$ for $u\left\llcorner F_{a}^{-1}(w)\right.$. For $g \in C^{\infty}\left(\mathbb{R}^{n}\right)$, it is well known that

$$
\sup _{S_{a}^{m}(1)}|g| \leq C\left(\int_{S_{a}^{m}(1)}|\nabla g|^{p}+|g|^{p} d H^{m}\right)^{1 / p},
$$

with $C=C(m, p)$, and by a simple scaling argument that

$$
\sup _{S_{a}^{m}(r)}|g| \leq C\left(\int_{S_{a}^{m}(r)} r^{p-m}|\nabla g|^{p}+r^{-m}|g|^{p} d H^{m}\right)^{1 / p} .
$$


Since $u_{k}\left\llcorner F_{a}^{-1}(w)\right.$ converges uniformly to $\mathbf{u} L F_{a}^{-1}(w)$ and strongly to $u_{w}$ in the sense of (4.4), applying (4.6) with $g$ replaced by $u_{k}$ yields (4.3).

Step 2. We will show that there exist a constant $C_{2}>0$ and an $H^{n-m_{-}}$ measurable set $E_{r} \subset I_{r}$ of positive $H^{n-m}$-measure such that

$$
\int_{F_{a}^{-1}(w)}|\nabla u|^{p}+\left|\frac{u-\bar{u}(a, r)}{r}\right|^{p} d H^{m} \leq C_{2} r^{m} \text { for each } w \in E_{r} .
$$

From the hypotheses that $a$ is an $L^{p}$-Lebesgue point for both $u$ and $|\nabla u|$ and that $\bar{u}(a)>0$, it follows that there exist positive numbers $R$ and $\kappa$ such that for $r \in(0, R)$ we have

$$
\bar{u}(a, r)>\kappa>0
$$

and

$$
\int_{B_{a}^{n}(r)}|\nabla u|^{p} d H^{n} \leq\left(|\overline{\nabla u}(a)|^{p}+1\right) H^{n}\left(B_{a}^{n}(r)\right) .
$$

Using Poincarè's inequality and (4.9), there exists $C_{1}=C_{1}(n, p)$ such that

$$
\begin{aligned}
\int_{B_{a}^{n}(r)}|u-\bar{u}(a, r)|^{p} d H^{n} & \leq C_{1} \int_{B_{a}^{n}(r)}|\nabla u|^{p} r^{p} d H^{n} \\
& \leq C_{1} \alpha_{n}\left(|\overline{\nabla u}(a)|^{p}+1\right) r^{n+p}
\end{aligned}
$$

where $\alpha_{n}$ is the volume of the unit ball in $\mathbb{R}^{n}$, and consequently,

$$
\int_{B_{a}^{n}(r)}\left|\frac{u-\bar{u}(a, r)}{r}\right|^{p} d H^{n} \leq C_{1} \alpha_{n}\left(|\overline{\nabla u}(a)|^{p}+1\right) r^{n} \text { for } r \in(0, R) .
$$

Employing the co-area formula, (4.10) and (4.9), we have for all $r \in(0, R)$,

$$
\begin{aligned}
\int_{I_{r}} \int_{F_{a}^{-1}(w)}|\nabla u|^{p} & +\left|\frac{u-\bar{u}(a, r)}{r}\right|^{p} d H^{m}(t) d H^{n-m}(w) \\
& =\int_{F_{a}^{-1}\left(I_{r}\right)}\left|J F_{a}\right|\left(|\nabla u|^{p}+\left|\frac{u-\bar{u}(a, r)}{r}\right|^{p}\right) d H^{n} \\
& \leq \int_{B_{a}^{n}(r+r \sqrt{n} / 2)}\left(|\nabla u|^{p}+\left|\frac{u-\bar{u}(a, r)}{r}\right|^{p}\right) d H^{n} \\
& \leq \alpha_{n}\left(1+\frac{\sqrt{n}}{2}\right)^{n}\left(C_{1}+1\right)\left(|\overline{\nabla u}(a)|^{p}+1\right) r^{n} .
\end{aligned}
$$

That is, setting $C_{2}=\alpha_{n}(1+\sqrt{n} / 2)^{n}\left(C_{1}+1\right)\left(|\overline{\nabla u}(a)|^{p}+1\right)$, we have

$$
\int_{I_{r}} \int_{F_{a}^{-1}(w)}|\nabla u|^{p}+\left|\frac{u-\bar{u}(a, r)}{r}\right|^{p} d H^{m} d H^{n-m}(w) \leq C_{2} r^{n} .
$$

Let $G(w)$ denote the inner integral in this expression, so that we have

$$
\int_{I_{r}} G(w) d H^{n-m}(w) \leq C_{2} r^{n},
$$

which establishes (4.7). 
Step 3. Finally, we will establish (ii) of our theorem. Since $E_{r} \subset I_{r}$, notice that for $w \in E_{r}, F_{a}^{-1}(w)$ is an $m$-sphere whose radius, $w_{1}=: \rho$, has the property that $r / 2 \leq \rho \leq 3 r / 2$. Thus, using (4.3) and (4.7), we obtain

$$
\begin{aligned}
\sup _{F_{a}^{-1}(w)} & \left|\frac{\mathbf{u}-\bar{u}(a, r)}{r}\right|^{p} \\
\leq & C \int_{F_{a}^{-1}(w)}\left(\rho^{p-m}\left|\nabla\left(\frac{u_{w}-\bar{u}(a, r)}{r}\right)\right|^{p}+\rho^{-m}\left|\frac{u_{w}-\bar{u}(a, r)}{r}\right|^{p}\right) d H^{m} \\
\leq & C\left(\frac{3}{2}\right)^{p} \rho^{-m} \int_{F_{a}^{-1}(w)}\left(\left|\nabla\left(u_{w}\right)\right|^{p}+\left|\frac{u_{w}-\bar{u}(a, r)}{r}\right|^{p}\right) d H^{m} \\
& \leq C\left(\frac{3}{2}\right)^{p} 2^{m} r^{-m} \int_{F_{a}^{-1}(w)}\left(\left|\nabla\left(u_{w}\right)\right|^{p}+\left|\frac{u_{w}-\bar{u}(a, r)}{r}\right|^{p}\right) d H^{m} \\
& \leq C_{2} C\left(\frac{3}{2}\right)^{p} 2^{m} .
\end{aligned}
$$

With $K:=\left(C_{2} C\left(\frac{3}{2}\right)^{p} 2^{m}\right)^{p}$, we have $\sup _{F_{a}^{-1}(w)}|\mathbf{u}-\bar{u}(a, r)| \leq K r$ for $w \in E_{r}$. This, along with (4.8), implies there exists $R_{0}>0$ such that $\mathbf{u}>0$ on $F_{a}{ }^{-1}(w)$ for $w \in E_{r}, 0<r<R_{0}$.

Theorem 4. Let $n \geq 3, n>m$ and $p>m \geq n-1-m \geq 1$. If $u$ is a non-zero element of $W^{1, p}\left(\mathbb{R}^{n}\right)$, then $u$ has a pair of linked spheres of dimensions $m$ and $n-1-m$ in its support.

Proof. If $u \in W^{1, p}\left(\mathbb{R}^{n}\right)$ is not identically zero, then there exists $a \in \mathbb{R}^{n}$ such that $a$ is an $L^{p}$-Lebesgue point for $u$ and $|\nabla u|$. We will assume without loss of generality, that $\bar{u}(a)>0$. Applying Theorem 3 we obtain $r_{0}>0$ and a Borel set $E_{r_{0}} \subset I_{r_{0}}$ of positive $H^{n-m}$-measure such that for $w \in E_{r_{0}} \subset \mathbb{R}^{n-m}$, $\mathbf{u}$ is continuous and positive everywhere on $F_{a}^{-1}(w)=S_{\left(a^{\prime}, w_{2}, \ldots, w_{n-m}\right)}^{m}\left(w_{1}\right)$. With a slight abuse of the notation introduced at the beginning of Section 4 , we let $w^{\prime \prime}:=\left(w_{2}, \ldots, w_{n-m}\right)$ so that we now have

$$
F_{a}^{-1}(w)=S_{\left(a^{\prime}, w^{\prime \prime}\right)}\left(w_{1}\right) .
$$

Let $W_{a}:=\bigcup_{w \in E_{r_{0}}} F_{a}^{-1}(w)$. Since $E_{r_{0}}$ is $H^{n}$-measurable and $J F_{a}=1$, we can appeal to the co-area formula to conclude that

$$
H^{n}\left(W_{a}\right)=\int_{E_{r_{0}}} H^{m}\left(F_{a}^{-1}(w)\right) d H^{n-m}(w)>0 .
$$

Note that $\mathbf{u}$ is defined and is positive at all points of $W_{a}$. For suitable $w \in W_{a}$, we will construct an $(n-m-1)$-sphere that will link with $S_{\left(a^{\prime}, w^{\prime \prime}\right)}^{m}\left(w_{1}\right)$ and that will lie in a "radial" $(n-m)$-plane emanating from $\left(a^{\prime}, w^{\prime \prime}\right)$ orthogonal to $Q^{-1}\left(w^{\prime \prime}\right)$. For this purpose define

$$
P: \mathbb{R}^{n} \backslash \bar{B}_{\left(a^{\prime}, w^{\prime \prime}\right)}^{n}\left(r_{0} / 2\right) \rightarrow S_{\left(a^{\prime}, w^{\prime \prime}\right)}^{m}(1) \quad \text { by } P(x)=\left(a^{\prime}+\frac{x^{\prime}-a^{\prime}}{\left|x^{\prime}-a^{\prime}\right|}, w^{\prime \prime}\right) .
$$

Observe that $P$ is locally Lipschitz and that $P^{-1}(\theta)$ is independent of $w$ for $\theta \in$ $S_{\left(a^{\prime}, w^{\prime \prime}\right)}^{m}(1)$. Proceeding as in the proof of Theorem 3 Step 1, with $F_{a}$ replaced by $P$, an application of the co-area formula yields that $u\left\llcorner P^{-1}(\theta) \in W^{1, p}\left(P^{-1}(\theta)\right)\right.$ for $H^{m}$-a.e. $\theta \in S_{\left(a^{\prime}, w^{\prime \prime}\right)}^{m}(1)$ and that $\mathbf{u}\left\llcorner P^{-1}(\theta)\right.$ is a $p$-quasicontinuous representative for $u\left\llcorner P^{-1}(\theta)\right.$; see (4.4) and (4.5). Since $H^{n}\left(W_{a}\right)>0$, the co-area formula also 
implies that $H^{n-m}\left(W_{a} \cap P^{-1}(\theta)\right)>0$ for $H^{m}$-a.e $\theta \in S_{\left(a^{\prime}, w^{\prime \prime}\right)}^{m}(1)$. Thus, for such $\theta$, there exists

$$
w \in W_{a} \cap P^{-1}(\theta)
$$

such that $w$ is a Lebesgue point for both $u\left\llcorner P^{-1}(\theta)\right.$ and $\nabla\left(u\left\llcorner P^{-1}(\theta)\right)\right.$. Since $H^{n-m}\left(W_{a} \cap P^{-1}(\theta)\right)>0$ and $\mathbf{u}\left\llcorner P^{-1}(\theta)>0\right.$ on $W_{a} \cap P^{-1}(\theta)$, it follows that we can also require $w$ to have been chosen so that

$$
\overline{u \mathrm{~L} P^{-1}(\theta)}(w)>0 .
$$

With $w$ determined by (4.11) and (4.12), it follows that $u \mathrm{~L} P^{-1}(\theta)$ satisfies the hypotheses of Theorem 3 (ii) with the ambient space $\mathbb{R}^{n}$ replaced by $P^{-1}(\theta)$ and with $F_{a}$ replaced by $D: P^{-1}(\theta) \rightarrow \mathbb{R}^{1}$, defined by $D(x)=\left|x-\left(a^{\prime}, w^{\prime \prime}\right)\right|$. Theorem 3 (ii) provides a number $0<\bar{r}<w_{1} / 2$ and a set $A \subset(\bar{r} / 2,3 \bar{r} / 2)$ of positive $H^{1}$ measure such that $\mathbf{u}\left\llcorner P^{-1}(\theta)\right.$ is defined and positive on each $D^{-1}(\rho), \rho \in A$. Thus we have that $\mathbf{u}>0$ on the $(n-m-1)$-sphere $D^{-1}(\rho)$ and $\mathbf{u}>0$ on the $m$-sphere $S_{\left(a^{\prime}, w^{\prime \prime}\right)}^{m}(r)$. These spheres are linked, since they are similar to the linked spheres (4.1) and (4.2).

Theorem 5. Let $h: \bar{B}^{k} \rightarrow \mathbb{R}^{n}$ be an embedding of the closed ball $\bar{B}^{k} \subset \mathbb{R}^{k+1}$ where $1 \leq k<n$ and $n \geq 3$. If $u \in W^{1, p}\left(\mathbb{R}^{n}\right), p>\left[\frac{k+1}{2}\right]$ and $\operatorname{spt} u \subset h\left(\bar{B}^{k}\right)$, then $u \equiv 0$.

Proof. First, assume $k$ is even, $k+1<n$, and by contradiction, suppose that $H^{n}(\operatorname{spt} u)>0$. Writing $x \in \mathbb{R}^{n}$ as $x=\left(x^{\prime}, x^{\prime \prime}\right)$ where $x^{\prime} \in \mathbb{R}^{k+1}$, recall that $Q: \mathbb{R}^{n} \rightarrow \mathbb{R}^{n-k-1}$ is defined as $Q(x):=x^{\prime \prime}$. Then we have $H^{k+1}\left(Q^{-1}\left(x^{\prime \prime}\right) \cap\right.$ spt $u)>0$ for all $x^{\prime \prime}$ in a set $E$ of positive $H^{n-k-1}$-measure and, as in (4.5), $u$ is a nonzero element of $W^{1, p}\left(Q^{-1}\left(x^{\prime \prime}\right)\right)$ for $H^{n-k-1}$-a.e. $x^{\prime \prime} \in E$. Redefine $E$ to include only such $x^{\prime \prime}$. For $x^{\prime \prime} \in E$, we employ Theorem 4 with $\mathbb{R}^{n}$ replaced by the $(k+1)$-dimensional affine space $Q^{-1}\left(x^{\prime \prime}\right)$ and $m$ replaced by $k / 2$ to conclude that $u \in W^{1, p}\left(Q^{-1}\left(x^{\prime \prime}\right)\right)$ contains a pair of linked spheres, both of dimension $k / 2$, in its support. Call these spheres $S_{1}$ and $S_{2}$. With $h$ as in the statement of our theorem, let $H:=h^{-1}\left\llcorner\left(Q^{-1}\left(x^{\prime \prime}\right) \cap h\left(\bar{B}^{k}\right)\right)\right.$; so $H$ is a homeomorphism of $\left(Q^{-1}\left(x^{\prime \prime}\right) \cap h\left(\bar{B}^{k}\right)\right)$ into $\mathbb{R}^{k}$. Since $S_{1}$ and $S_{2}$ are linked spheres in $\left(Q^{-1}\left(x^{\prime \prime}\right) \cap h\left(\bar{B}^{k}\right)\right)$ and since $H$ is a homeomorphism, it follows from Definition 1 that $H\left(S_{1}\right)$ and $H\left(S_{2}\right)$ are linked in $\mathbb{R}^{k}$, which contradicts Theorem 2

The above proof is easily modified and simpler for the case $k+1=n$. A similar argument holds when $k$ is odd.

\section{REFERENCES}

[AH96] D. R. Adams and L. I. Hedberg, Function Spaces and Potential Theory, Springer-Verlag, Berlin, 1996. MR 97j:46024

[Bes50] A. Besicovitch, Parametric Surfaces, Bulletin of the American Mathematical Society, 56 (1950), pp. 288-296. MR 12:168e

[BG98] T. Bagby and P.M. Gauthier, Note on the support of Sobolev functions, Canadian Math. Bulletin 41 (3) (1998), pp. 257-260. MR 99g:46037

[Gage81] M. Gage, On Gehring's Linked Sphere Problem, American Journal of Mathematics, 103, no. 3. (June, 1981), pp. 437-443. MR 82j:52024

[Fed59] H. Federer, Curvature measures, Trans. Amer. Math. Soc. 93 (3) (1959), pp. 418-491. MR 22:961

[GT83] D. Gilbarg and N. S. Trudinger, Elliptic Partial Differential Equations of Second Order, Second Edition, Springer-Verlag, 1983. MR 86c:35035

[Hir76] M. W. Hirsch, Differential Topology, Springer-Verlag, New York, 1976. MR 56:6669 
[MZ97] Jan Malý and W. P. Ziemer, Fine regularity of solutions of elliptic partial differential equations, Math. Surveys and Monographs, Amer. Math. Soc., Providence, RI, 1997. MR 98h:35080

[Mas91] W. S. Massey, A Basic Course in Algebraic Topology, Springer-Verlag, New York, 1991. MR 92c:55001

[Pol72] J. C. Polking, A Leibnitz formula for some differentiation operators of fractional order, Indiana Univ. Math. J. 21 (1972) pp. 1019-1029. MR 47:7414

[Pon87] S. P. Ponomarev, On the $N$-property of homeomorphisms of the class $W_{p}^{1}$, Siberian Math. Journal 28 (1987) pp. 291-298. MR 88i:26031

[Rol76] D. Rolfson, Knots and Links, Publish or Perish, Berkeley, California, 1976. MR 58:24236

[Zie89] W. P. Ziemer, Weakly Differentiable Functions, Springer-Verlag, New York, 1989. MR 91e:46046

Department of Mathematics, California State University Long Beach, Long Beach, CALifornia 90840-1001

E-mail address: wziemer@csulb.edu

URL: http://www.csulb.edu/depts/math 